\title{
On performance sensitivity of urban transportation networks
}

\author{
A. Di Febbraro \& N. Sacco \\ Department of Machines, Energy Systems, and Transportation, \\ University of Genoa, Italy Via Montallegro 1, 16145, Genoa, Italy
}

\begin{abstract}
The continuous increase in traffic, intended as interacting vehicles using a common infrastructure, leading to traffic congestion whenever mobility demand exceeds the infrastructure capacity itself, has put into evidence many needs in transportation. In this framework, the choice of the optimal solution is a difficult problem to tackle with, especially when uncertainties are taken into account. Therefore, the main effort of this paper is to define a selection criteria which helps in resource planning and in decision making, based on a sensitivity matrix computed for an a-priori chosen transportation performance index.
\end{abstract}

Keywords: urban traffic networks, resources planning, performance index, sensitivity matrix.

\section{Introduction}

The continuous increase in traffic, intended as interacting vehicles using a common infrastructure, leading to traffic congestion whenever mobility demand exceeds the infrastructure capacity itself, has put into evidence many needs in transportation. On the other hand, the limited economic and spatial resources are major reasons why the expansion of the already existing civil infrastructures has to be carefully evaluated. This is one of the reasons for which, in the last decades, communication and information technologies for the optimisation of the existing resources had found many applications in transportation.

In effect, nowadays it is evident that the request of an efficient, safe, secure and less polluting transportation has to be faced generally by means of integrated solutions, including both the expansion of the infrastructures and the introduction ITS devices for traffic optimisation. 
Then, such a problem results to be quite difficult to tackle with and needs to be considered from a general point of view, where the interactions between the proposed civil and ITS solutions are taken into account at a time. Moreover, the problem becomes more difficult when several different solutions have to be compared, and especially when the actual and the future performances of the transportation system are considered. Other elements of great importance are the uncertainties of the transportation system parameters and the non-deterministically predictable variations of the mobility demand which typically grows but, in some cases, may decrease, for instance when modal shift are promoted.

Therefore, the main effort of this paper is to define a selection criteria, which helps in resource planning and in decision making, based on sensitivity matrices computed for a-priori chosen transportation performance indices.

The paper is organised as follows. In Section 2 a formal definition of the considered transportation system is given. Then, in the following Section 3, the sensitivity matrix and the best configuration selection criteria are discussed. Finally, Section 4, and Section 5 are devoted to a description of the selected case study and to the conclusions, respectively.

\section{The formal approach}

In the following sections a suitable definition of urban transportation network and the sensitivity index are introduced.

\subsection{Main definitions}

The definition of Urban Transportation Network (UTN) here given has the aim of pointing out the interaction between the transportation capacity of the infrastructures and the mobility demand. Then, in order to keep general the proposed approach, in the proposed definition the civil structures (roads, intersections, etc.), and the technological devices (such as traffic light controller, Variable Message Signs (VMS), etc.) are considered separately.

Then, an UTN may be defined as the triple

$$
U T N=\left\{\mathcal{C}, \mathcal{I}, \mathcal{K}, \mathcal{M}_{\mathcal{O D}}\right\}
$$

where $\mathcal{C}=\left\{C_{j}, j=1, \ldots, \mathrm{J}_{\mathrm{C}}\right\}$ is the set of the centroids representing the different zones of the considered area, whereas $\mathcal{I}=\left\{I_{j}, j=1, \ldots, \mathrm{J}_{\mathrm{I}}\right\}$ and $\mathcal{K}=\left\{K_{j}, j=\right.$ $\left.1, \ldots, \mathrm{J}_{\mathrm{K}}\right\}$ are the set of the civil infrastructures and of technological devices "working" in the considered area, respectively. Finally, the term $\mathcal{M}_{\mathcal{O D}} \in \mathbb{R}^{J_{C}, J_{C}}$ is the origin-destination matrix, whose generic element $\mathcal{M}_{\mathcal{O D}}(i, j)$ in a non-negative real number representing the mobility demand from the centroid $i$ towards the centroid $j$. In this definition, the elements representing the mobility demand with origin and destination in the same centroid are neglected, that is $\mathcal{M}_{\mathcal{O D}}(j, j)=0$, $\forall j=1, \ldots, J_{C}$.

For what concerns the the performances of a UTN, they depend on the network elements and their interactions in a way frequently difficult to analytically describe, 
especially when large networks are considered. In effects, this is the reason why simulation is typically used in transportation network projecting and transportation planning.

In this framework, defining an analytical procedure to help to choose, among the different possible improvements and modifications, the best set of interventions that may be implemented at a time and also fulfilling the budget constraints, result to be a interesting problem to solve. In order to do so, in this paper a sensitivity index of the performances of a transportation network will be described.

Then, consider a set of $m_{i}$ different network improvements which may be applied in an UTN. As said, these changes may be adopted in both the elements defining the transportation supply or network capacity, and may consist, for instance, of the introduction of new traffic controllers or of the modification of the viability and the mobility demand, perhaps due to the modal-shift towards public or railway transportation.

Afterwards, given $m_{i}$ different possible improvements, then there are $M_{c}=2^{m_{i}}$ possible configurations in which one or more of the improvements are implemented at a time. For instance, if there are $m_{i}=2$ possible network improvements, such as a new structure like a new road, and a new traffic manager like a traffic light controller, then there are $M_{c}=2^{2}=4$ configurations:

$s_{1}$ : this is the present situation, that is without any improvements;

$s_{2}$ : in this configuration, only the built of the new road is considered;

$s_{3}$ : in this configuration, only the implementation of the new traffic manager is considered;

$s_{4}$ : in this configuration, both the structural and technological improvements are considered at a time.

Then, in this framework, it is possible to define the vector

$$
s=\left[\begin{array}{llll}
s_{1} & s_{2} & \ldots & s_{M_{c}}
\end{array}\right]
$$

in which each element is a sequence $s_{i}=\left\{s_{i}(k), k=1,2, \ldots, m_{i}\right\}, s_{i}(k) \in\{0,1\}$, $i=1,2, \ldots, M_{c}$, whose $k^{\text {th }}$ element $s_{i}(k)$ is set to one $\left(s_{i}(k)=1\right)$ or to zero $\left(s_{i}(k)=0\right)$, whether the $k^{\text {th }}$ possible intervention is considered or not in the $i^{\text {th }}$ configuration, respectively. With this definition, it results that the sequences

$$
s_{1}=\overbrace{\left\{\begin{array}{lll}
0 & \ldots & 0
\end{array}\right\}}^{m_{i}} \text { and } s_{M_{c}}=\overbrace{\left\{\begin{array}{lll}
1 & \ldots & 1
\end{array}\right\}}^{m_{i}},
$$

represent the present network without any intervent and the configuration characterised by the presence of all the intervets, respectively. Then, coming back to the above example, the considered configurations are mapped into the sequences

$$
s_{1}=\left\{\begin{array}{ll}
0 & 0
\end{array}\right\}, \quad s_{2}=\left\{\begin{array}{ll}
0 & 1
\end{array}\right\}, \quad s_{3}=\left\{\begin{array}{ll}
1 & 0
\end{array}\right\}, \quad \text { and } \quad s_{4}=\left\{\begin{array}{ll}
1 & 1
\end{array}\right\} .
$$


For what concerns the origin-destination matrix, being $J_{C}$ the number of centroids in the considered UTN, it is possible to define the vector

$$
O D=\left[\begin{array}{llll}
o d_{1} & o d_{2} & \ldots & o d_{n_{O D}}
\end{array}\right]
$$

of the couples origin-destination, being $n_{O D}=J_{C}^{2}-J_{C}$ is the total number of different couples. Note that in this definition the couples with the same origin and destination centroid, that is those corresponding to the diagonal elements $\mathcal{M}_{\mathcal{O D}}(j, j)=0$, are not considered.

Note that some changes in a UTN may lead to new definitions or to the suppression of centroids. This might come, for instance, when a new parking area is built. In these cases, the dimension of the vector $O D$ changes accordingly. Nevertheless, in this present paper it is assumed that the vector $O D$ has fixed dimensions, i.e., in other words, that the number of centroids was constant.

\subsection{Performance index definition}

Consider now a general performance index $\phi\left(s_{i}, O D\right)$, computed for a fixed UTN configuration $s_{i}$, and at a "nominal" value of the demand vector $O D$. Then, it is possible to define the performance vector

$$
\Phi(s, O D)=\left[\begin{array}{llll}
\phi\left(s_{1}, O D\right) & \phi\left(s_{2}, O D\right) & \ldots & \phi\left(s_{M_{c}}, O D\right)
\end{array}\right]
$$

which gathers the values assumed by the parameter $\phi\left(s_{i}, O D\right)$ computed for all the possible configurations and by assuming the nominal demand $O D$.

Such a vector is able to give information about the best configurations to realise. In fact, depending of the chosen performance index, the best configuration coincides with the one corresponding to the minimum or to the maximum element of the vector (4), that is

$$
i^{*}=\arg \min _{i} \phi\left(s_{i}, O D\right)
$$

or

$$
i^{*}=\arg \max _{i} \phi\left(s_{i}, O D\right),
$$

respectively.

For instance, if $\phi\left(s_{i}, O D\right)$ gives the mean transit time in the UTN, then the best configuration is given by (5). On the other hand, if $\phi\left(s_{i}, O D\right)$ represents the mean vehicle speed, then then the best configuration is given by (6).

Note that the performances parameter $\phi\left(s_{i}, O D\right), \forall i=1, \ldots, M_{c}$, may be a suitable combination of different indices which takes into account various aspects of the considered problem at a time, such as the mean travel time, the delay time, some pollution indices, the realisation costs, or a cost-benefit ratio. 


\section{Sensitivity of the vector $\Phi(s, O D)$}

As said, the above defined vector $\Phi(s, O D)$ does not give information concerning the influence of the mobility demand uncertainty which, on the other hand, can not be neglected. Altough any new infrastructure is normally projected by assuming a "increased" demand $O D^{\prime}>O D$, it is not guaranteed that, due to the complex interactions among all the elements of the UTN, the whole system real performances result to be the best one under real conditions.

In order to cope with this problem, each element $\phi\left(s_{i}, O D\right)$ of the performance vector $\Phi(s, O D)$ may be associated to a row of a sensitivity matrix $S$, whose generic element $S_{i, j}$, computed with respect to the configuration $s_{i}$, and to the demand $o d_{j}$, is given by the relation

$$
S_{\Phi}(i, j)=\frac{\frac{\partial \phi\left(s_{i}, O D\right)}{\phi\left(s_{i}, O D\right)}}{\frac{\partial o d_{j}}{o d_{j}}}, \quad i=1, \ldots, M_{c}, \quad j=1, \ldots, n_{O D}
$$

The ratio in the Eq. (7) expresses the relative deviation of the performance index $\phi\left(s_{i}, O D\right)$ computed for the configuration $s_{i}$ with respect its nominal value, compared with the relative variation of the term $o d_{j}$. In order to compute such a value, Eq. (7) may be written as

$$
S_{\Phi}(i, j)=\frac{\partial \phi\left(s_{i}, O D\right)}{\partial o d_{j}} \cdot \frac{o d_{j}}{\phi\left(s_{i}, O D\right)}, \quad i=1, \ldots, M_{c}, \quad j=1, \ldots, n_{O D}
$$

in which:

- the term partial derivative express how amplified or reduced is the variation of the performances when the demand $o d_{j}$ varies;

- the term $o d_{j}$ is the "nominal" demand for which the network improvements are projected;

- the term $\phi\left(s_{i}, O D\right)$ is the "nominal" performance, that is the network performances computed at the nominal demand computed for the configuration $s_{i}$.

Then, once computed all the terms of the sensitivity matrix, it is possible to choose the configuration with the greatest robustness to the changes in $O D$. In fact, it corresponds to the row which fulfils the minimum-norm criteria, that is

$$
i^{*}=\arg \min _{i}\left\|S_{\Phi}(i,:)\right\|_{p}, \quad i=1, \ldots, M_{c}
$$

where $S_{\Phi}(i,:)$ is the $i^{\text {th }}$ row of the sensitivity matrix $S_{\Phi}$, while the subscript $p=$ $1,2, \infty$ indicates the selected norm.

For what concerns the meaning of the three considered norms in (9), it is worth saying that

1. when $p=\infty$, the norm coincides with the greatest element of the argument vector, that is

$$
\left\|S_{\Phi}(i,:)\right\|_{\infty}=\max _{j}\left|S_{\Phi}(i, j)\right|, \quad j=1, \ldots, n_{O D} .
$$


Such a norm may be used to point out the most critical situation, but does not takes into account the overall performance of the net;

2. when $p=2$, the norm coincides with the euclidean norm

$$
\left\|S_{\Phi}(i,:)\right\|_{2}=\sqrt{\sum_{j=1}^{n_{O D}}\left[S_{\Phi}(i, j)\right]^{2}} .
$$

In this case, due to the squared values, the greatest elements of the row $i$ weight more than the littlest ones. In particular, the sensitivity values in absolute value less than one tend to be reduced, while the terms greater than one tend to be amplified;

3. when $p=1$, the norm is given by the relation

$$
\left\|S_{\Phi}(i,:)\right\|_{1}=\sum_{j=1}^{n_{O D}}\left|S_{\Phi}(i, j)\right|
$$

In this case, all the elements of the vector $S_{\Phi}(i,:)$ are taken into account without any amplification or attenuation. This norm is useful when distortions in the sensitivity matrix have to be avoided.

Altough the above defined index $\Phi(s, O D)$ and the relevant sensitivity matrix $S_{\Phi}$ gives useful information about the best configuration to choose, they often cannot be easily computed due to the lack of analytical traffic models capable to take into account all the UTN aspects. In order to tackle with this problem, in the following section a simple procedure to compute the elements of the sensitivity matrix by means of a simulation approach is described.

\subsection{Computing the sensitivity elements}

In order to compute the sensitivity values of Eq. (8), consider the (symmetric) derivative approximation

$$
\frac{\partial \phi\left(s_{i}, O D\right)}{\partial o d_{j}} \simeq \frac{\phi\left(s_{i}, O D+0.5 \cdot \Delta_{j}\right)-\phi\left(s_{i}, O D-0.5 \cdot \Delta_{j}\right)}{\Delta_{j}},
$$

where

$$
\begin{aligned}
& 1^{\text {st }} \quad \ldots \quad j^{\text {th }} \quad \ldots \quad n_{O D}^{\text {th }} \\
& \downarrow \downarrow \downarrow \downarrow \downarrow \downarrow \\
& \Delta_{j}=\left[\begin{array}{lllllll}
0 & \ldots & \ldots & \delta & \ldots & 0 &
\end{array}\right],
\end{aligned}
$$

is a vector in which all the elements are null, but the $j^{\text {th }}$ one that assumes a suitable small value $\delta$.

Then, with the aim of evaluating the sensitivity matrix, it is necessary to compute the values of $\phi(\cdot)$ in $\left(s_{i}, O D\right)$, and in $\left(s_{i}, O D \pm 0.5 \cdot \Delta_{j}\right), i=1, \ldots, M_{c}$, $j=1, \ldots, n_{O D}$. Then, the number $\#_{o p}$ of operation necessary to computed all the elements of the sensitivity matrix is $\#_{o p}=M_{c}\left(2 n_{O D}+1\right) R$, being $R$ the number 
of different simulation which guarantees a suitable statistical significance value of each parameter $\phi(\cdot)$ (see, for instance [1], for further details).

Moreover, altough the total number of simulations may be high, many traffic simulators allow to automatically repeat the simulations, thus leaving to the network designer the only duty of defining the different configurations $s_{i}$. Finally, on the other hand, the number of possible intervents is often small, thus limiting the relevant configurations.

\section{Case study}

In this section, in order to provide a numerical example for the proposed procedure, a case study is described. Consider the simple UTN consisting of a four-way intersection represented in Fig. 1.A which is characterised by $J_{C}=4$ centroids representing the north, east, south, and west zones of surrounding urban area.

As regards the origin-destination matrix $\mathcal{M}_{\mathcal{O D}}$, has $J_{C}^{2}=16$ elements but, due to the intersection layout of Fig. 1.A the relevant vector $O D$ may be reduced to the six non null elements representing the admissible flows crossing the intersection, thus becoming

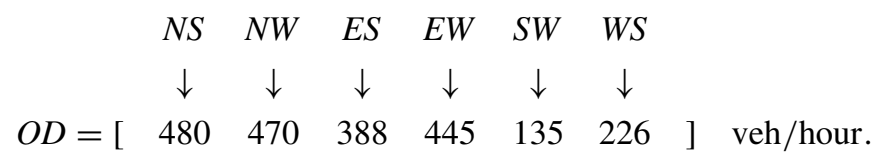

Then, consider $m_{i}=2$ possible intervents, consisting of the improvement of some of the in coming road of the considered intersection and of the introduction of an optimised traffic responsive traffic light controller, respectively. The resulting $M_{c}=4$ configurations are

$s_{1}:$ it is the nominal configuration, that is the one without interventions;

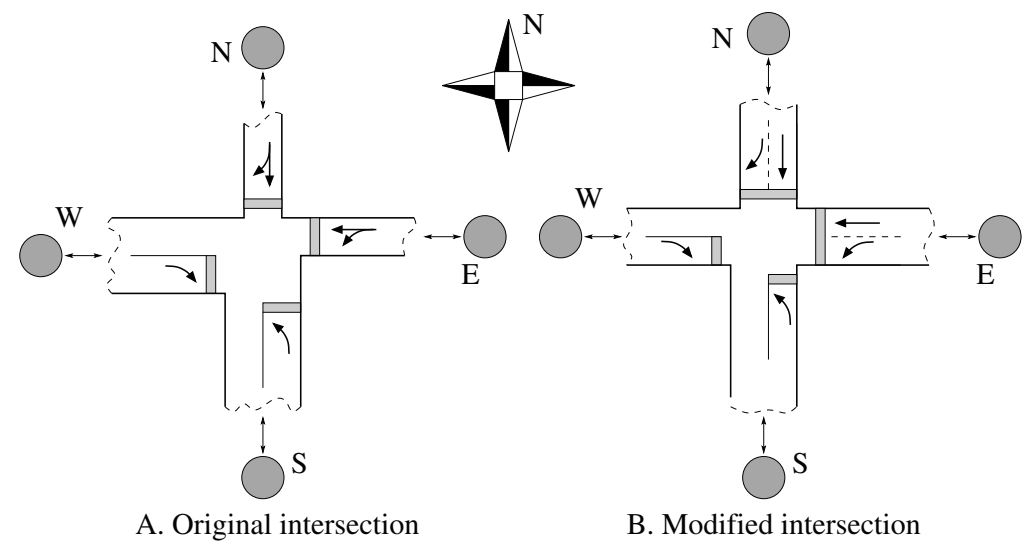

Figure 1: A simple UTN. 
$s_{2}$ : in this configuration only the road improvement is considered;

$s_{3}$ : in this configuration only the traffic light controller in considered;

$s_{4}$ : this is the complete configuration, with both the interventions.

The resulting improbed layout of configurations $s_{2}$ and $s_{4}$ is depicted in Fig. 1.B.

As regards the performances, the parameter vector $\Phi(s, O D)$ gathers the mean queue length

$$
\phi\left(s_{i}, o d\right)=\frac{1}{n_{Q}} \sum_{h=1}^{n_{Q}} Q_{h}^{i}(t),
$$

being $T$ the simulation duration, $Q_{h}(t)$ is the length of the queue at the incoming direction $h$ at time $t$ and in configuration $s_{i}$, and $n_{Q}$ the number of different queues in the different configurations, that is, $n_{Q}=4$ in configurations $s_{0}$ and $s_{2}$, and $n_{Q}=4$ in configurations $s_{1}$ and $s_{3}$.

Then, by means of a hybrid Petri net model, based on the methodology developed in [2], the parameter vector $\Phi(s, O D)$ may be computed and results to be

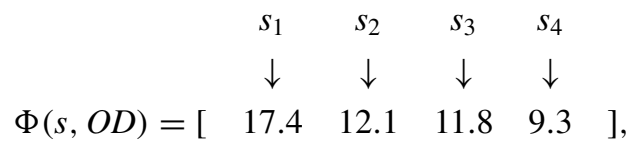

while the relevant sensitivity matrix is

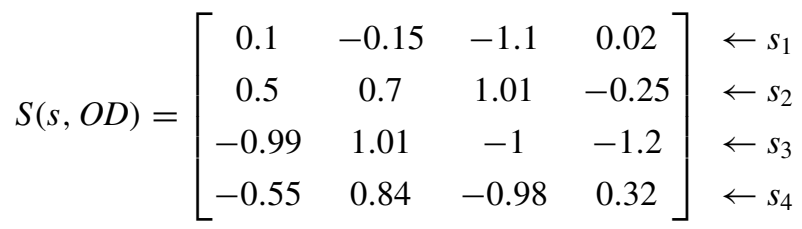

Then, the norms of each row of the sensitivity matrix are reported in Tab. 1, where it is possible to note that:

- configuration $s_{1}$ is the more stable. This is mainly due to the fact that, with the present layout and traffic light plan, the intersection is always highly congested, so that the demand variations do not significantly influence the UTN performances;

- configuration $s_{3}$ is the less robust. This means that, while the relevant performance is fairly good with respect to the others, it can be reached only with a perfectly known or estimated demand. Nevertheless, such a drawback should be resolved by introducing a more sophisticated on-line traffic light controller able to well estimating the real traffic conditions at any time;

- configurations $s_{2}$ and $s_{4}$ have almost equal $p$-norms, $\forall p$. It means that the intervention which really changes the UTN performances, contemporaneously guaranteeing the stability of the network, is the road enlargement. On the other hand, the performance of these "stable" solutions are enhanced 
Table 1: Norms of the sensitivity matrix of the study case.

\begin{tabular}{llll}
\hline$s_{i}$ & $\left\|s_{i}\right\|_{\infty}$ & $\left\|s_{i}\right\|_{2}$ & $\left\|s_{i}\right\|_{1}$ \\
\hline$s_{1}$ & 1.11 & 1.37 & 1.1 \\
$s_{2}$ & 1.35 & 2.46 & 1.01 \\
$s_{3}$ & 2.11 & 4.2 & 1.2 \\
$s_{4}$ & 1.44 & 2.69 & 0.98 \\
\hline
\end{tabular}

by the introduction of responsive traffic light controller, which improve the performances without affecting the robustness significantly.

Then, it is possible to state that from the point of view of the selected performance parameter $\phi\left(s_{i}, O D\right)$, the best configuration for the considered case study results to be $s_{4}$, corresponding to the implementation of both the proposed interventions. In effect, such a configuration guarantees the best reduction of the mean queue length, while being characterised by a robustness almost equal to the minimum one, in all the considered norms.

\section{Conclusions}

In this paper, a methodology for the selection of a robust set of improving intervents in a UTN has been described. More precisely, once defined the general framework, the sensitivity matrix has been described and the relevant row norm has been discussed. In the end of the paper, a simple case study has been described, with the aim of clarifying the presented methodology. Work is in progress on extending the present methodology to the evaluation of the effects of unpredictable events, such as, for instance, accidents.

\section{References}

[1] Law, A.M. \& Kelton, W., Simulation Modeling and Analysis. McGraw-Hill, $2^{\text {nd }}$ edition, 1991.

[2] Di Febbraro, A. \& Sacco, N., On modelling urban transportation networks via hybrid Petri nets. Control Engineering Practice, Special issue: Analysis and Design of Hybrid Systems, 12(10), pp. 1225-1239, 2004. 